\title{
Determinantes do desmame precoce no distrito noroeste de Campinas
}

\author{
Early weaning determinants in a district \\ of Campinas, Brazil
}

Cíntia Cristina de Almeida VOLPINI ${ }^{1}$

Erly Catarina MOURA²

RE S U M O

\section{Objetivo}

Investigar as características do desmame precoce no distrito Noroeste da cidade de Campinas, SP.

\section{Métodos}

Durante a Campanha Nacional de Vacinação de 8 de junho de 2001, estudo transversal foi conduzido junto a 385 crianças menores de dois anos (10\% do atendimento). O responsável pela criança foi questionado sobre características sociodemográficas e assistenciais, idade e motivo da introdução de outros alimentos e do desmame. Considerou-se desmame precoce a interrupção total da oferta de leite materno antes de seis meses de idade. A associação entre desmame e fatores causais e/ou de risco foi investigada por correlação bivariada. Para determinar se a associação persistia, regressão logística foi executada para identificação do(s) melhor(es) preditor(es) do desmame. Para a identificação das causas alegadas de desmame precoce, utilizou-se o teste de diferença de proporções.

\section{Resultados}

O desmame precoce atingiu $63,6 \%$ das crianças. O tempo de estudo materno se mostrou associado ao desmame precoce, conforme teste " $\mathrm{t}$ " $(p=0,04)$. Não houve diferença quanto à idade, estado marital e trabalho materno, número de pessoas na família, renda, tipo de assistência e sexo da criança. A regressão logística indicou a idade de introdução de leite não materno $(p<0,0001)$ como preditor do desmame. Os motivos alegados para o desmame precoce foram: o fato de o leite ter secado; rejeição pelo bebê; trabalho materno; doença materna; dores ao amamentar; problemas na mama e doença da criança.

\section{Conclusão}

Os resultados apontaram a introdução precoce de leites e fórmulas como preditor do desmame precoce.

Termos de indexação: aleitamento materno, bem-estar da criança, desmame precoce, leite humano.

\footnotetext{
1 Bolsista de Iniciação Científica PIBIC-CNPq. Faculdade de Medicina, Centro de Ciências da Vida, Pontifícia Universidade Católica de Campinas. Campinas, SP, Brasil.

2 Núcleo de Pesquisas Epidemiológicas em Nutrição e Saúde, Faculdade de Saúde Pública, Universidade de São Paulo. Avenida Dr. Arnaldo, 715, 01246-904, São Paulo, SP, Brasil. Correspondência para/Correspondence to: E.C.MOURA. E-mail: <erlycm@usp.br>.
} 


\section{A B S T R A C T}

\section{Objective}

To investigate determinants of early weaning in Northwest of Campinas, Sao Paulo state, Brazil.

\section{Methods}

During the National Immunization Day on June 8th, 2001, a survey was conducted among 385 children less than 2 years old (10\% of attendance). The responsible for the child was interviewed about social, demographic, and health assistance characteristics, age, and reasons for the introduction of other foods to the child and its weaning. The complete interruption of breast-feeding before the age of 6 months was considered as early weaning. The associations between weaning and cause and/or risk factors were investigated by bivariate correlation. To check if the association was maintained, logistic regression was run to identify the best predictors for early weaning. The proportions' difference test was performed to identify alleged reasons for early weaning.

\section{Results}

Early weaning affected $63.6 \%$ of the surveyed children. The education level of the mother was associated to early weaning, according to t-test $(\mathrm{p}=0.04)$. There was no association between early weaning and mother's age, marital status or occupation, family density, income, kind of health assistance, and gender of the child. Logistic regression indicated time of artificial milk or formula introduction as predictor for early weaning $(\mathrm{p}<0.0001)$. The alleged reasons for early weaning were: decrease of maternal milk production, baby's refusal to breast-feed, mother's occupation, pain during breast-feeding, breast injuries, and sickness of either mother or baby.

\section{Conclusion}

The results indicated the early introduction of milk or formulas as a predictor for early weaning.

Indexing terms: breast feeding, child welfire, early weaning, milk human.

\section{N T R O D U ÇÃ O}

Nos últimos anos, a política de saúde da criança no Brasil tem priorizado as ações de promoção, proteção e apoio ao aleitamento natural, sendo essa uma estratégia fundamental para reduzir a mortalidade infantil no país e para melhorar a saúde das crianças brasileiras'.

Apesar das abundantes evidências científicas $^{2-6}$ da superioridade do leite materno sobre outros tipos de leite, ainda é baixo o número de mulheres que amamentam seus filhos de acordo com as recomendações da Organização Mundial de Saúde e do Ministério da Saúde7, que estabelecem o aleitamento materno de forma exclusiva até os seis meses de vida e complementar até 24 meses de idade ou mais.

No Brasil ${ }^{7}$, a prevalência de amamentação aumentou na última década, passando de 49\% aos seis meses de idade, na década de 80 , para $60 \%$ na década de 90 . Porém, o incremento nos índices de aleitamento materno não foi homogêneo em todo o país, sofrendo variações significativas de acordo com o local e as características socioeconômicas da população estudada. Por esse motivo, é imprescindível conhecer as características locais do padrão de aleitamento natural, a fim de avaliar os fatores de risco para o desmame precoce e contribuir para o planejamento em saúde na formulação de ações educativas e de suporte que favoreçam o aumento da prática da amamentação.

Com esse intuito, o presente estudo teve como objetivo conhecer as características do desmame precoce entre os menores de dois anos de idade na população residente no distrito Noroeste de Campinas.

\section{MÉ T O D OS}

Este estudo transversal foi desenvolvido no distrito de saúde Noroeste do município de 
Campinas, contando com a aprovação do Comitê de Ética da Pontifícia Universidade Católica de Campinas (PUC-Campinas). Campinas é uma das principais cidades do Estado de São Paulo, localizando-se a cem quilômetros ao Noroeste da capital do estado. Conta com uma população de cerca de um milhão de habitantes, e divide-se em cinco grandes áreas geográfico-administrativas: Norte, Sul, Leste, Sudoeste e Noroeste. A região Noroeste tem aproximadamente 150 mil habitantes e é representativa do município no que se refere à população residente. O distrito Noroeste comporta sete unidades básicas de saúde (atenção primária), alguns ambulatórios de especialidade públicos e privados (atenção secundária) e o hospital universitário da Pontifícia Universidade Católica de Campinas (atenção terciária).

A amostra aleatória sistemática foi composta de $10 \%$ das crianças menores de dois anos de idade que compareceram aos postos de vacinação durante o primeiro dia da Campanha Nacional de Vacinação de 2001, que contou com uma cobertura de $93 \%$. Os responsáveis pelas crianças foram submetidos a entrevista por alunos dos cursos de medicina e nutrição, treinados para esse fim. Para os acompanhantes dessas crianças foi aplicado questionário pré-codificado, contendo informações sobre características sociodemográficas (idade, anos de estudo, estado civil e trabalho materno; número de pessoas e renda mensal per capita da família; sexo da criança em estudo) e assistenciais (realização, local e orientação sobre amamentação no pré-natal; tipo, local e orientação sobre amamentação no parto; local e orientação sobre amamentação na puericultura), além da idade da criança quando da introdução de outros alimentos, que não o leite materno, na dieta infantil. Considerou-se desmame precoce quando a oferta de leite materno foi totalmente interrompida durante o primeiro semestre de vida. Para aquelas mães que desmamaram a criança precocemente foi questionado o motivo da introdução de outro alimento na dieta infantil, considerado como causa alegada para o desmame precoce. As causas foram classificadas em educacionais (relacionadas à falta de informação/orientação), sociais (relacionadas à pressão social externa) e fisiológicas (relacionadas a problema biológico possivelmente impeditivo do aleitamento materno).

A análise dos dados foi processada utilizando-se o programa Statistical Package for the Social Sciences e os resultados foram descritos utilizando-se o teste " $t$ " na comparação das médias das variáveis contínuas e o teste qui-quadrado na comparação da distribuição das variáveis categóricas entre as crianças com e sem desmame precoce, considerando-se nível de significância de $5 \%$. Para determinar se a associação persistia, regressão logística foi executada, incluindo-se todas as variáveis associadas ao nível de $p \leq 0,05$, comparando-se os dois grupos na busca da identificação do(s) melhor(es) preditor(es) do desmame precoce. Para a identificação das causas alegadas de desmame precoce, utilizou-se o teste de diferença de proporções com intervalo de confiança de $95 \%$.

\section{RES ULTA DOS}

Foram entrevistados os responsáveis de 385 crianças, sendo que $160(41,6 \%)$ ainda eram amamentadas e $225(58,4 \%)$ já haviam sido desmamadas. Os dados analisados neste trabalho se referem apenas às 225 crianças desmamadas. Dessas, 143 (63,6\%) foram desmamadas precocemente, ou seja, antes dos seis meses de idade. A idade de desmame precoce foi $76 \pm 50$ dias e de desmame após seis meses foi $278 \pm 115$ dias (cerca de nove meses).

Das variáveis estudadas, apenas o tempo de estudo materno se mostrou associado ao desmame precoce, isto é, mães com menor tempo de estudo tendem a desmamar antes dos seis meses $(p=0,04)$, sendo que o risco de desmame precoce de uma mãe que estudou menos de oito anos foi o dobro $(\mathrm{OR}=2,0 ; 1,1<\mathrm{IC}<3,7)$ daquela que estudou oito anos ou mais. Em média, as 
mulheres estudaram 8,2 $\pm 3,2$ anos, sendo que $42,1 \%$ estudaram menos do que oito anos (Tabela 1).

Quanto à idade, identificaram-se 34 $(15,1 \%)$ mães adolescentes (de 16 a 19 anos de idade). A maioria das mães apresentou estado marital estável $(79,6 \%)$ e não trabalhava após o nascimento da criança (58,3\%). Das mães que trabalhavam, a média de horas trabalhadas por dia foi de 8,2 ( $D P=2,0$ ) e 58,9\% eram registradas ( $n=56$ de um total de 95 informações). Não houve diferença quanto à idade de desmame da criança entre as trabalhadoras formais e as informais.

Em geral, as famílias das crianças desmamadas eram compostas por 4,3 pessoas e recebiam mensalmente cerca de $\mathrm{R} \$ 238,00$ per capita, isto é, US\$101.00. Das crianças, 49,8\% eram do sexo feminino e 50,2\% do masculino.

No que concerne à assistência à saúde (Tabela 2), observou-se que 98,2\% das mães realizaram o pré-natal, sendo a maioria $(44,2 \%)$ na rede básica de saúde e um número significativo
(41,0\%) na rede privada. Uma mãe não soube informar se houve pré-natal por se tratar de adoção. A maioria das mães $(58,2 \%)$ referiu não ter recebido orientação sobre a amamentação durante o pré-natal. Daquelas 94 que receberam orientação, 66 (70,2\%) referiram ter sido o médico o responsável pela orientação, $35(37,2 \%)$ o pessoal de enfermagem e $15(16,0 \%)$ outros profissionais da saúde; apenas $15(16,0 \%)$ receberam estímulo de familiares ou amigos. $\mathrm{Na}$ rede básica de saúde, $44,8 \%$ das mães referiram que a orientação foi dada pelo pessoal de enfermagem, 43,7\% pelos médicos e 7,5\% por outros profissionais.

No total dos partos, 50,0\% foram cirúrgicos. A prevalência de partos cirúrgicos foi maior na Maternidade de Campinas (64,9\%, isto é, 48 de 74$)$, seguida por outros hospitais $(62,1 \%$, isto é, 41 de 66); o hospital universitário da PUC-Campinas teve $27,7 \%$ (23 de um total de 83 ) dos partos realizados por cesárea. A realização dos partos foi maior no hospital local, o Hospital Universitário

Tabela 1. Média (M) e desvio-padrão (DP) ou freqüências (número e porcentagem) das características sociodemográficas das crianças desmamadas. Campinas, 2001.

\begin{tabular}{|c|c|c|c|c|c|c|}
\hline \multirow{3}{*}{ Variáveis sociodemográficas } & \multicolumn{4}{|c|}{ Desmame precoce } & & \\
\hline & \multicolumn{2}{|c|}{$\operatorname{Sim}(n=143)$} & \multicolumn{2}{|c|}{ Não $(n=82)$} & \multicolumn{2}{|r|}{ Total } \\
\hline & M & DP & M & DP & M & DP \\
\hline Idade materna (anos) & 25,1 & 6,3 & 25,9 & 5,8 & 25,4 & $\pm \quad 6,1$ \\
\hline Estudo materno (anos) & 7,9 & 3,3 & 8,8 & 3,1 & 8,2 & 3,2 \\
\hline Número de pessoas na família & 4,3 & 1,4 & 4,4 & $\pm \quad 2,5$ & 4,3 & $\pm \quad 1,9$ \\
\hline \multirow[t]{2}{*}{ Renda mensal per capita (reais) } & 224,0 & $\pm 208,0$ & 263,0 & $\pm 238,0$ & 238,0 & $\pm 220,0$ \\
\hline & \multicolumn{4}{|c|}{ Desmame precoce } & & \\
\hline \multirow[t]{2}{*}{ Variáveis sociodemográficas } & \multicolumn{2}{|c|}{$\operatorname{Sim}(n=143)$} & \multicolumn{2}{|c|}{ Não $(n=82)$} & \multicolumn{2}{|r|}{ Total } \\
\hline & $n$ & $\%$ & $n$ & $\%$ & $\mathrm{n}$ & $\%$ \\
\hline \multicolumn{7}{|l|}{ Estado civil materno } \\
\hline Estável & 110 & 76,9 & 69 & 84,1 & 179 & 79,6 \\
\hline instável & 33 & 23,1 & 13 & 15,9 & 46 & 20,4 \\
\hline \multicolumn{7}{|l|}{ Trabalho materno após o parto } \\
\hline Sim & 58 & 40,8 & 35 & 43,2 & 93 & 41,7 \\
\hline Não & 84 & 59,2 & 46 & 56,8 & 130 & 58,3 \\
\hline \multicolumn{7}{|l|}{ Sexo da criança em estudo } \\
\hline Feminino & 69 & 48,3 & 43 & 52,4 & 112 & 49,8 \\
\hline Masculino & 74 & 51,7 & 39 & 47,6 & 113 & 50,2 \\
\hline
\end{tabular}


da PUC-Campinas $(37,2 \%)$, seguido pela Maternidade de Campinas (33,2\%) e por outros hospitais (29,5\%). A grande maioria das mães $(61,8 \%)$ referiu não ter recebido nenhuma orientação sobre amamentação durante sua internação para a resolução do parto. Do total de 83 mulheres que receberam orientação, 43,4\% eram da PUC-Campinas, 36,5\% da Maternidade e $34,8 \%$ de outros hospitais. Das 112 mulheres que tiveram parto cirúrgico, 40 (35,7\%) receberam orientação sobre amamentação, contra 46 $(41,1 \%)$ das 112 com parto normal. Dos 83 partos realizados no hospital local, as orientações foram dadas por médicos a 38 (45,8\%) mulheres, pela enfermagem a $34(41,0 \%)$ e por outros profissionais a $10(12,5 \%)$.

Observa-se, ainda, que $51,4 \%$ das crianças utilizavam a rede básica de saúde para o acompanhamento de rotina, $32,4 \%$ os serviços privados e $16,2 \%$ os hospitais públicos. Apenas $29,8 \%$ das mães referiram ter recebido orientação durante a puericultura. Os hospitais públicos orientaram 31 (46,3\%) mães, os serviços privados 23 (34,3\%) e a rede básica apenas 17 (25,4\%). As orientações foram ministradas principalmente pelos médicos (54,7\%), seguidos pelo pessoal de enfermagem $(40,4 \%)$ e outros profissionais da saúde $(10,2 \%) ; 7,6 \%$ das mães receberam

Tabela 2. Freqüência (número e porcentagem) das características assistenciais das crianças desmamadas. Campinas, 2001.

\begin{tabular}{|c|c|c|c|c|c|c|}
\hline \multirow{3}{*}{ Variáveis assistenciais } & \multicolumn{4}{|c|}{ Desmame precoce } & \multirow{2}{*}{\multicolumn{2}{|c|}{ Total }} \\
\hline & \multicolumn{2}{|c|}{$\operatorname{Sim}(n=143)$} & \multicolumn{2}{|c|}{ Não $(n=82)$} & & \\
\hline & $\mathrm{n}$ & $\%$ & $\mathrm{n}$ & $\%$ & $\mathrm{n}$ & $\%$ \\
\hline \multicolumn{7}{|c|}{ Realização de pré-natala } \\
\hline Sim & 139 & 97,9 & 81 & 98,8 & 220 & 98,2 \\
\hline Não & 3 & 2,1 & 1 & 1,2 & 4 & 1,8 \\
\hline \multicolumn{7}{|l|}{ Local do pré-natala,b } \\
\hline Hospitais universitários & 22 & 15,9 & 10 & 12,7 & 32 & 14,8 \\
\hline Rede básica de saúde & 63 & 45,7 & 33 & 41,8 & 96 & 44,2 \\
\hline Rede privada & 53 & 38,4 & 36 & 45,5 & 89 & 41,0 \\
\hline \multicolumn{7}{|c|}{ Orientação sobre amamentação no pré-natal } \\
\hline Sim & 61 & 42,7 & 33 & 40,2 & 94 & 41,8 \\
\hline Não & 82 & 57,3 & 49 & 59,8 & 131 & 58,2 \\
\hline \multicolumn{7}{|l|}{ Tipo de parto ${ }^{a}$} \\
\hline Normal & 74 & 52,1 & 38 & 46,3 & 112 & 50,0 \\
\hline Cirúrgico & 68 & 47,9 & 44 & 53,7 & 112 & 50,0 \\
\hline \multicolumn{7}{|l|}{ Local do partoc } \\
\hline Outro hospital & 45 & 31,9 & 21 & 25,6 & 66 & 29,6 \\
\hline Hospital PUC-Campinas & 53 & 37,6 & 30 & 36,6 & 83 & 37,2 \\
\hline Maternidade & 43 & 30,5 & 31 & 37,8 & 74 & 33,2 \\
\hline \multicolumn{7}{|c|}{ Orientação sobre amamentação no parto } \\
\hline Sim & 55 & 38,5 & 31 & 37,8 & 86 & 38,2 \\
\hline Não & 88 & 61,5 & 51 & 62,2 & 139 & 61,8 \\
\hline \multicolumn{7}{|l|}{ Local da puericulturad } \\
\hline Rede básica & 78 & 55,7 & 36 & 43,9 & 114 & 51,4 \\
\hline Rede privada & 42 & 30,0 & 30 & 36,6 & 72 & 32,4 \\
\hline Hospital público & 20 & 14,3 & 16 & 19,5 & 36 & 16,2 \\
\hline \multicolumn{7}{|c|}{ Orientação sobre amamentação na puericultura } \\
\hline Sim & 42 & 29,4 & 25 & 30,5 & 67 & 29,8 \\
\hline Não & 101 & 70,6 & 57 & 69,5 & 158 & 70,2 \\
\hline
\end{tabular}

${ }^{\mathbf{a}}$ sem informação= 1 (adotada); ${ }^{\mathbf{b}}$ sem informação= 8 (1 adotada e 7 não sabiam); ' sem informação= 2 (1 adotada e 1 não sabia); ${ }^{\mathbf{d}}$ sem informação = 3 (não sabiam). 
orientação de familiares ou amigos. Na rede básica, $44,8 \%$ das mães relataram ter recebido orientação de médicos, $38,6 \%$ do pessoal da enfermagem e $9,1 \%$ de outros profissionais. No ambulatório de pediatria do hospital universitário, as 36 orientações foram dadas em sua maioria pelos médicos ( $n=17,47,2 \%$ ) e pelo pessoal de enfermagem ( $n=17,47,2 \%)$, os outros profissionais foram responsáveis pela orientação de apenas duas $(11,8 \%)$ das mulheres.

Encontrou-se associação entre desmame precoce e idade de introdução de alimentos (Tabela 3), sendo que o chá foi o mais precocemente introduzido nos dois grupos de crianças desmamadas (60 e 95 dias de idade, em média, respectivamente). A ordem de introdução de alimentos nos dois grupos foi semelhante, à exceção do leite não materno, que ocupou o segundo lugar no grupo das crianças desmamadas precocemente e o último no grupo das crianças desmamadas após seis meses.

A regressão logística, incluindo todas as variáveis listadas na Tabela 3, mostrou que apenas a idade de introdução de leite não materno se manteve associada ao desmame precoce $(p<0,0001)$, significando que, nessa população, a

Tabela 3. Idade média (em dias) de introdução de alimentos das crianças desmamadas. Campinas, 2001.

\begin{tabular}{|c|c|c|c|c|c|c|}
\hline \multirow{3}{*}{ Alimentos } & \multicolumn{5}{|c|}{ Desmame precoce } & \multirow{3}{*}{$p$} \\
\hline & \multicolumn{2}{|c|}{$\operatorname{Sim}(n=143)$} & \multicolumn{3}{|c|}{ Não $(n=82)$} & \\
\hline & $M$ & $\pm \mathrm{DP}$ & $M$ & \pm & $\mathrm{DP}$ & \\
\hline Chá & 60 & \pm 52 & 95 & \pm & 80 & 0,005 \\
\hline Leite não materno & 73 & $\pm \quad 51$ & 208 & \pm & 111 & $<0,001$ \\
\hline Água & 75 & $\pm \quad 50$ & 117 & \pm & 59 & $<0,001$ \\
\hline Suco & 112 & $\pm \quad 45$ & 137 & \pm & 61 & 0,005 \\
\hline Frutas & 130 & \pm 64 & 158 & \pm & 54 & 0,002 \\
\hline Papinha & 138 & \pm 49 & 160 & \pm & 42 & 0,001 \\
\hline
\end{tabular}

Tabela 4. Distribuição (\%) das razões alegadas pela mãe para o desmame. Campinas, 2001.

\begin{tabular}{|c|c|c|c|}
\hline \multirow{2}{*}{ Causas educacionais } & \multicolumn{2}{|c|}{ Desmame precoce } & \multirow{2}{*}{ IC } \\
\hline & $\operatorname{Sim}(n=143)$ & Não $(n=82)$ & \\
\hline O fato de o leite ter secado & 28,70 & 2,40 & 18,20 a 34,40 \\
\hline Rejeição do bebê & 21,70 & 6,10 & 5,70 a 25,50 \\
\hline Leite fraco & 5,60 & 1,20 & $-0,04$ a $\quad 8,80$ \\
\hline Choro do bebê & 5,60 & 2,40 & $-1,80$ a 8,20 \\
\hline Falta de orientação & 4,20 & 1,20 & $-1,10$ a 7,00 \\
\hline Dores ao amamentar & 3,50 & - & 0,50 a 6,50 \\
\hline Problemas na mama & 2,80 & - & 0,10 a $\quad 5,50$ \\
\hline Nervosismo materno & 2,10 & 1,20 & $-2,40$ a 4,20 \\
\hline \multicolumn{4}{|l|}{ Sociais } \\
\hline Trabalho materno & 13,30 & 1,20 & 6,10 a 18,10 \\
\hline Conveniência & 9,10 & 6,10 & $-4,00$ a 10,00 \\
\hline Orientação profissional da saúde & 5,60 & 4,90 & $-5,30$ a 6,70 \\
\hline Conselho de terceiros & 0,70 & - & $-0,70$ a 2,10 \\
\hline \multicolumn{4}{|l|}{ Fisiológicas } \\
\hline Doença materna & 7,70 & - & 3,30 a 12,10 \\
\hline Doença da criança & 2,80 & - & 0,10 a $\quad 5,50$ \\
\hline Cirurgia na mama & 2,10 & - & $-0,30$ a 4,50 \\
\hline Abandono materno & 1,40 & - & $-0,50$ a 3,30 \\
\hline
\end{tabular}


introdução de leite não materno foi o preditor do desmame antes de seis meses de idade. A inclusão da variável anos de estudo materno, no modelo analítico, não alterou a relação.

Questionadas sobre as causas do desmame precoce (Tabela 4), 93 (65,0\%) das 143 mães acompanhantes as relacionaram a problema de ordem educacional, $34(23,8 \%)$ social e 15 $(10,5 \%)$ fisiológica, havendo uma ou mais causas relacionadas pelas mães. Os motivos alegados para o desmame precoce foram: o fato de o leite ter secado, rejeição do bebê, trabalho materno, doença materna, dores ao amamentar, problemas na mama e doença da criança, de acordo com intervalo de confiança de 95,0\%.

\section{DISCUSS ÃO}

Este estudo se refere a apenas uma região do município de Campinas, mas os resultados obtidos podem ser considerados para outras regiões da cidade, uma vez que há relativa homogeneidade no que se refere à estrutura dos serviços de saúde, à qualificação dos recursos humanos na área e às características socioeconômicas e culturais da população. Em adição, os dados obtidos fornecem subsídios ao Distrito de Saúde local para o planejamento em saúde visando à promoção do aleitamento materno. Todavia, viéses de memória no que se refere principalmente à idade de desmame e à introdução de alimentos na dieta infantil são mais freqüentes em estudos retrospectivos, como este. Datas de acontecimentos mais antigos tendem a ser arredondadas para meio mês ou mês inteiro, gerando uma informação não precisa, ainda que aproximada, da real data de desmame e introdução de alimentos. De qualquer modo, mesmo considerando esses viéses, a idade em que houve o desmame, considerada como a data de interrupção completa da oferta de leite materno, é muito baixa na população estudada. Outro fator limitante se refere à causa alegada de desmame precoce, que tende a se alterar à medida que há maior aprofundamento na relação profissional-cliente, como já apontavam Réa \& Cukier $^{8}$.

Neste estudo, quanto às condições sociodemográficas da população estudada, a variável escolaridade materna se mostrou associada ao desmame precoce, isto é, quanto maior o tempo de escolaridade da mãe, maior a duração do aleitamento natural. Outros estudos ${ }^{9,10}$ também constataram o aumento da prevalência do aleitamento exclusivo entre as mulheres com maior escolaridade. Segundo Kummer et al. ${ }^{11}$, essa tendência provavelmente se deva à maior valorização dos benefícios da prática do aleitamento materno nas classes mais favorecidas econômica e culturalmente. Os autores levantam a hipótese de que as mulheres mais instruídas estão em fase de valorização do aleitamento materno exclusivo, enquanto as mulheres menos esclarecidas ainda não foram sensibilizadas.

Observou-se, também, neste estudo, que não houve diferença na idade de desmame entre as mães trabalhadoras e as mães não trabalhadoras, o que demonstra que o trabalho materno não é causa direta do desmame precoce nessa população, embora o desejo de trabalhar tenha se associado à interrupção do aleitamento natural. Esse achado vai contra outros estudos ${ }^{8,10}$ que apontaram o trabalho materno como causa de desmame. Todavia, estudos mais recentes ${ }^{6,12}$ apontam uma maior prevalência de amamentação entre as mulheres trabalhadoras. É necessário nos inquéritos sobre causas de desmame investigar se a mãe estava, de fato, trabalhando, ou se tinha apenas o desejo/necessidade de fazê-lo. Os fatores de risco associados ao desmame precoce parecem estar muito mais relacionados à falta de informação sobre o manejo da lactação durante a gestação, o parto e a puericultura do que ao trabalho materno, como apontam Carlon-Aviles et al. ${ }^{13}$.

O fato de mais da metade das mães não terem recebido orientação sobre amamentação no pré-natal é um dado preocupante. Harada et al. ${ }^{14}$ encontraram $57,5 \%$ das mães sem orientação; Giugliani et al. ${ }^{15}$, 47,0\% no pré-natal e 48,0\% na maternidade. Tal fato aponta para a 
falha no acompanhamento das mães pelos profissionais de saúde durante o pré-natal, assim como no pós-parto, períodos fundamentais para a orientação das mães sobre as técnicas corretas e os benefícios do aleitamento materno ${ }^{16}$. 0 profissional de saúde responsável pela assistência à mulher requer não apenas conhecimentos sobre amamentação, mas também necessita de habilidades clínicas e de aconselhamento. O aconselhamento em amamentação implica ajudar a mãe a tomar decisões de forma empática, saber ouvir, dar apoio e sugestões, além de desenvolver a confiança. É importante que as mães se sintam encorajadas a prosseguir com o aleitamento natural. Apesar de formalmente convencidos das vantagens e benefícios da amamentação, são poucos os profissionais de saúde que se dedicam a esclarecer gestantes e puérperas sobre a importância do aleitamento exclusivo.

Nesta pesquisa, o médico foi o informante e orientador mais lembrado pelas mães, independente do local de atendimento (setor privado, hospitais públicos e rede básica). Tal menção talvez se deva ao fato de que o médico ainda é a figura de maior legitimidade dentro do setor da saúde. É necessário ressaltar a pequena participação da equipe de enfermagem na orientação sobre amamentação, uma vez que esses profissionais são peças fundamentais na assistência à saúde, devendo ajudar, apoiar e incentivar o processo de lactação a fim de promover o completo entendimento e assimilação da prática do aleitamento materno.

Assim como apontado por Toma \& Monteiro ${ }^{17}$, neste estudo, os hospitais públicos foram os que mais prestaram informações às mães (46,4\%), seguidos pelos hospitais privados (33,3\%). Práticas facilitadoras da amamentação tendem a ser encontradas com maior freqüência em hospitais públicos, principalmente nos hospitais universitários, talvez pelo fato de estarem mais comprometidos com a educação e a ciência.

Observa-se que as mães que desmamaram precocemente tendem a justificar o desmame principalmente com motivos de ordem educacional, como o fato de o leite ter secado $(28,7 \%)$, rejeição do bebê $(21,7 \%)$, presença de dores ao amamentar (3,5\%) e problemas na mama (2,8\%); seguidos pelos de ordem social, como desejo de retorno ao trabalho (13,3\%); e depois pelos de ordem fisiológica, como doença materna $(7,7 \%)$ e doença do bebê $(2,8 \%)$. O desmame por opção (conveniência) não se caracterizou como motivo para o desmame precoce, nem o choro do bebê, a falta de orientação ou o nervosismo materno. Esses achados estão de acordo com os relatados por Harada et al. ${ }^{14}$, em estudo com crianças menores de seis meses de idade. As autoras identificaram como causas principais de desmame a redução do leite, o trabalho materno e a recusa da criança.

Através da regressão logística, a única variável que se manteve associada ao desmame precoce foi a idade em que se iniciou a introdução de leite não materno. Outros estudos também demonstraram que a introdução de fórmulas e, conseqüentemente, da mamadeira em idade precoce faz com que haja diminuição da freqüência das mamadas, do tempo de sucção das mamas e da duração da lactação ${ }^{18,19}$. Mais uma vez, reforça-se o papel dos profissionais da saúde, aos quais cabe a obrigação de não prescrever fórmulas ou leites artificiais sem necessidade e de informar às mães sobre a importância da amamentação.

Concluindo, a introdução precoce de leites ou fórmulas foi o preditor do desmame precoce na população estudada. É indubitável a necessidade de se investir em orientação materna, em treinamento dos profissionais, em uso dos meios de comunicação e em modificação das rotinas hospitalares na busca do incentivo à amamentação, uma vez que o aleitamento é uma habilidade que precisa ser resgatada e uma prática que precisa ser apoiada pelos profissionais da saúde e por toda a sociedade. Dar o peito é um ato produtivo, exclusivo da mulher, que necessita ser mais valorizado.

\section{REFER Ê N CIAS}

1. Araújo MFM. Situação e perspectivas do aleitamento materno no Brasil. In: Carvalho RM, 
Tamez RN. Amamentação: bases científicas para a prática profissional. Rio de Janeiro: Guanabara Koogan; . p.1-9.

2. Janke JR. The incidence, benefits and variables associated with breastfeeding: implications for practice. Nurs Pract. 1993; 18(6):22-3,28,31-2.

3. Giugliani ERJ. O Aleitamento materno na prática clínica. J Pediatr. 2000; 21:238-52.

4. Victora CG, Vaughan JP, Lombardi C, Fuchs SMC, Gigante LP, Smith PG, et al. Evidence for protection by breast-feeding against infant deaths from infectious diseases in Brazil. Lancet. 1987; 8(8554):319-22.

5. Von Kries R, Koletzko B, Sauerwald T, Von Mutius E. Does breast-feeding protect against childhood obesity? Adv Exp Med Biol. 2000; 478:29-39.

6. Wagner $\mathrm{CL}$, Wagner MT, Hulsey TC. Factors influencing a mother's decision to breastfeed. Adv Exp Med Biol. 2000; 478:435-6.

7. Brasil. Ministério da Saúde. Secretaria de Política de Saúde. Guia alimentar para crianças menores de 2 anos. Brasília: MS; 2002.

8. Réa MF, Cukier R. Razões de desmame e de introdução de mamadeira: uma abordagem alternativa para seu estudo. Rev Saúde Pública. 1988; 22(3):184-91.

9. Labrada MCP, Rio MV, González RMG, Reyes WG. Factores maternos asociados a la duración de la lactancia materna en Santos Suárez. Rev Cubana Med Gen Integr. 1999; 15:397-402.

10. Bulk-Bunschoten AM, van Bodegom S, Reerink JD, Pasker-de Jong PC, Graat CJ. Reluctance to continue breastfeeding in The Netherlands. Acta Pediatr. 2001; 90(9):1047-53.

11. Kummer SC, Giugliani ERJ, Susin LO, Folletto JL, Lermen NR, Wu VYJ, et al. Evolução do padrão de aleitamento materno. Rev Saúde Pública. 2000; 34(2):143-8.
12. Awang $H$, Salleh ALH. Determinants of breastfeeding duration in peninsular Malaysia. J Public Health. 2000; 12(2):102-6.

13. Carlon-Aviles MG, Arroyos MAC, Hernandez-Bojorquez RE, Medina-Molina BA, Armenta GS, Chavez MLO, et al. Práctica de amamantamiento en madres trabajadoras y no trabajadoras que recibieron orientación sobre lactancia materna exclusiva. Rev Mex Puericultura y Pediatría. 2000; 8:8-10.

14. Harada MJCS, Peterlini MAS, Scardonelli R, Dias EC. Fatores associados ao desmame precoce e aleitamento misto em crianças internadas em uma enfermaria de pediatria. Acta Paul Enf. 1999; 12:27-34.

15.Giugliani ER, Issler RM, Justo EB, Deffrin CF, Hartmann RM, Carvalho NM. Risk factors for early termination of breast feeding in Brazil. Acta Paediatr. 1992; 81:484-7.

16.Abrão ACFV, Barros SMO, Almeida AM. Desmame precoce: estudos das causas em crianças de 0 a 6 meses de idade, Dourados-MS. Acta Paul Enf. 1997; 10:30-9.

17.Toma TS, Monteiro CA. Avaliação da promoção do aleitamento materno nas maternidades públicas e privadas do Município de São Paulo. Rev Saúde Pública. 2001; 35(5):409-14.

18. Hörnell A, Hofvander Y, Kylberg E. Solids and formula: Association with pattern and duration of breastfeeding. Pediatrics [serial on the Internet]. 2001; 107(3):[about 38p]. Available from: http:// www.pediatrics.org/cgi/content/full/107/3/e38

19. Marques NM, Lira PIC, Lima MC, Silva NL, Batista Filho $\mathrm{M}$, Huttly SRA, et al. Breastfeeding and early weaning practices in Northeast Brazil: A longitudinal study. Pediatrics [serial on the Internet]. 2001; 107(4):[about 66p]. Available from: http://www.pediatrics.org/cgi/content/ full/ 107/4/e66

Recebido para publicação em 26 de março de 2003 e aceito em 29 julho de 2005. 